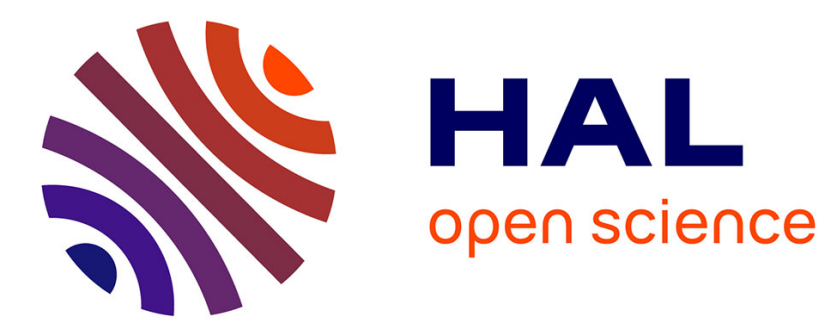

\title{
Some perspectives in electron momentum spectroscopy experiments
}

\author{
A. Bawagan
}

\section{To cite this version:}

A. Bawagan. Some perspectives in electron momentum spectroscopy experiments. Journal de Physique IV Proceedings, 1993, 03 (C6), pp.C6-175-C6-185. 10.1051/jp4:1993617 . jpa-00251702

\section{HAL Id: jpa-00251702 https://hal.science/jpa-00251702}

Submitted on 1 Jan 1993

HAL is a multi-disciplinary open access archive for the deposit and dissemination of scientific research documents, whether they are published or not. The documents may come from teaching and research institutions in France or abroad, or from public or private research centers.
L'archive ouverte pluridisciplinaire HAL, est destinée au dépôt et à la diffusion de documents scientifiques de niveau recherche, publiés ou non, émanant des établissements d'enseignement et de recherche français ou étrangers, des laboratoires publics ou privés. 


\title{
Some perspectives in electron momentum spectroscopy experiments
}

\author{
A.D.O. BAWAGAN \\ Ottawa-Carleton Chemistry Institute, 1125 Colonel By Drive, Carleton University, Ottawa, Ontario \\ K1S 5B6, Canada
}

\begin{abstract}
The basic challenges of electron momentum spectroscopy (EMS) as it has guided our research efforts at Carleton University are outlined. These challenges are: (a) Are the spectroscopic factors obtained in EMS equivalent to the spectroscopic factors observed in photoelectron spectroscopy? (b) Can experimental momentum profiles be related to the chemical reactivity of complex molecular systems? (c) Are the basic assumptions of $(e, 2 \mathrm{e})$ reaction theory valid for complex molecular systems? Insights based on our efforts to address these questions are presented.
\end{abstract}

\section{Introduction}

The major experimental observables in electron momentum spectroscopy (EMS) are the binding energy spectrum and the experimental momentum profile (XMP) of energy-selected states. Excellent surveys of the EMS literature up to 1988 are available $[1,2]$ so the present contribution will deal with more current developments with special focus on research developments at Carleton University. Although the EMS experimental observables are also available from other physico-chemical techniques such as photoelectron spectroscopy (PES) and Compton scattering, the subtle differences between the EMS observables and PES and Compton observables are oftentimes not appreciated $[3,4]$ if not misunderstood. The absence of a clear understanding of these differences has hindered the full development of EMS as a physico-chemical technique. At the very least, EMS provides binding energy spectra (ie. ionic state spectroscopic factors) and experimental momentum profiles complementary to those provided by other techniques. These information provided by EMS and PES will provide a more detailed understanding of the electronic structure of atoms, molecules and other complex chemical aggregates. Nonetheless the continued progress of EMS towards becoming a routine physico-chemical technique, as envisioned by its originators $[5,6,7]$, depend critically on the successful resolution of this and other outstanding challenges.

\section{Challenges in electron momentum spectroscopy}

I have ventured to outline some of the basic questions in EMS as it has guided our research efforts at Carleton. It is not meant to be an exhaustive or authoritative list nor an indication that these questions are more important than others. The basic questions are:

(a) Are the spectroscopic factors obtained in EMS equivalent to the spectroscopic factors observed in photoelectron spectroscopy? 
(b) Can experimental momentum profiles be related to the chemical reactivity of complex molecular systems?

(c) Are the basic assumptions of $(\mathrm{e}, 2 \mathrm{e})$ reaction theory valid for complex molecular systems?

Rather than providing an answer to each of these questions, I will try to address each question by way of example. That is, I will provide an update of our ongoing research efforts at Carleton with these questions in mind.

\subsection{Spectroscopic factors in EMS and PES}

An interesting historical account of the controversy between spectroscopic factors obtained in EMS and PES is provided by Brion et al. [8]. The controversy centers on the apparent differences between the Ar $3 s^{-1}$ satellite $^{1}$ intensities observed in EMS [9,10] and PES [11,12]. Current understanding however seem to suggest that these differences are due to the different ionization mechanisms in photoionization and electron impact ionization. These differences were first discussed by Khiefets [13] and McCarthy [3]. This is best illustrated by the following relation [4,13-15],

$$
\tilde{S}_{i}=S_{i}\left(1+\frac{\epsilon_{i}-E_{0}}{\Delta}\right)^{2}
$$

where $\tilde{S}_{i}$ and $S_{i}$ are the spectroscopic factors obtained in PES and EMS, respectively. $\epsilon_{i}$ is the exact energy of the ion state $i, E_{0}$ is the Hartree-Fock energy of the ion ground state and $\Delta$ is the average energy of two-hole-one-electron states. Equation 1 indicates that the PES spectroscopic factors are not the "true" spectroscopic factors and, in fact, can never approach the true spectroscopic factors $\left(S_{i}\right)$ even if the photon energy is varied. The only possibility is by accident, that is, whenever $\left(\epsilon_{i}-E_{0}\right)$ is negligible. That this is in fact the case is corroborated by recent studies by Khiefets and Amusia [15] and Braidwood et al. [16] on the spectroscopic factors of the Xe $5 s^{-1}$ satellites. In the case of Xe $5 s^{-1}$ satellites, $\left(\epsilon_{i}-E_{0}\right)$ is small and the satellite intensities obtained in EMS and PES are fairly similar in stark contrast to the case for $\mathrm{Ar} 3 s^{-1}$ satellites.

One of the factors to consider in comparing spectroscopic factors in EMS and PES is the larger energy resolution in EMS $\left(\geq 1.0 \mathrm{eV}\right.$ fwhm) relative to photoelectron spectroscopy using $\mathrm{Al} K_{\alpha}$ radiation $(\sim 0.5 \mathrm{eV}$ fwhm) or synchrotron radiation $(\sim 0.2 \mathrm{eV} \mathrm{fwhm})$. The energy resolution becomes a very important factor especially in the accurate determination of spectroscopic factors of closely spaced satellite or correlation states. In particular, a re-analysis [17] of previously reported EMS Ar $3 s^{-1}$ binding energy spectra of Leung and Brion [9] and McCarthy and Weigold [10] indicated that the reported differences between spectroscopic factors obtained in EMS and PES can be reconciled if energy resolutions and detailed fine structure are taken into account. It should be noted that this type of re-analysis does not contradict Eqn. 1 but rather provide a more realistic assessment of the error limits of previous EMS studies [9,10]. Clearly, a high energy resolution EMS study would be most helpful and progress have been made towards this end by Weigold and co-workers $[16,18]$.

Are spectroscopic factors in EMS and PES equivalent? I would like to approach this question from a different angle. Since 1989 we have initiated a research program investigating the correlation states of medium sized molecular systems using synchrotron photoelectron spectroscopy at Synchrotron Radiation Center (Aladdin) [19-22]. Careful calibration studies have been made so that accurate satellite intensities can be extracted from the synchrotron PES spectra. As a benchmark system we have chosen the ethylene molecule which is the simplest conjugated molecular system. Of particular interest is the correlation state at $27.4 \mathrm{eV}$ binding energy which has been previously observed by PES

\footnotetext{
1"Satellite" state is oftentimes a misleading term since there are many cases when the distinction between "parent" peak and "satellite" peak is not possible. The term "correlation state" is also used to refer to these states and reflects more the mechanism by which they arise.
} 


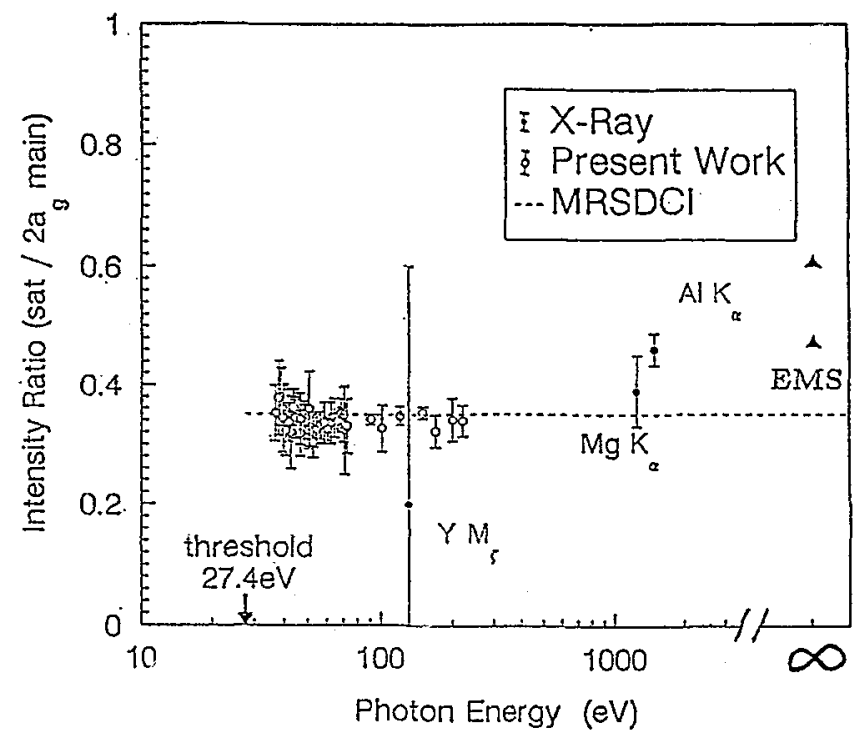

Figure 1: Photon energy dependence of the satellite $/ 2 a_{g}^{-1}$ main intensity ratios of ethylene [21] derived from synchrotron PES (open circles), previous X-ray measurements (solid circles) and previous EMS measurements (solid triangles) [24,25]. Dashed line is the predicted satellite/main intensity ratio from an MRSDCI calculation [26].

[23] and EMS $[24,25]$. Figure 1 shows the transmission corrected satellite $/ 2 a_{g}^{-1}$ main peak intensity ratio obtained from a series of synchrotron PES studies $[21,22]$ with an energy resolution of better than $0.2 \mathrm{eV}$ fwhm. The EMS satellite $/ 2 a_{g}^{-1}$ main intensity ratios obtained by Dixon et al. [24] and Coplan et al.[25] are shown at the "infinite" photon energy scale. Also shown are the calculated spectroscopic factors from the "cluster" of correlation states around $27.4 \mathrm{eV}$ obtained from accurate multireference singles and doubles configuration interaction (MRSDCI) calculations [26]. Even more sophisticated MRSDCI calculations involving a 196-GTO basis set indicated that the calculated intensity ratio is converged at a ratio of 0.35 [22] similar to that shown in Fig. 1. The important point to note is the quantitative agreement obtained between synchrotron PES spectroscopic factors and the spectroscopic factors calculated using very accurate MRSDCI wavefunctions. Is this fortuitous? Is this agreement due to a negligible $\left(\epsilon_{i}-E_{0}\right)$ energy difference?

For the ethylene satellite this energy difference is $\sim 0.5 \mathrm{eV}$ which can be compared with the Xe $5 s^{-1}$ satellite $\left(5 s^{2} 5 p^{4}\left({ }^{1} D\right) 5 d\left({ }^{2} S\right)\right)$ which is $\sim 2 \mathrm{eV}$ and the energy difference for the Ar $3 s^{-1}$ satellite $\left(3 s^{2} 3 p^{4}\left({ }^{1} D\right) 3 d\left({ }^{2} S\right)\right.$ ) which is $\sim 4 \mathrm{eV}$. Thus it would appear that the ethylene satellite at 27.4 $\mathrm{eV}$ is an ideal test case for the Khiefets-Amusia theory [4]. If the satellite/ $2 a_{g}^{-1}$ main intensity ratios obtained from the two previous EMS studies are averaged we obtain a ratio of 0.54 which is markedly different from the intensity ratios obtained from synchrotron PES and those predicted by MRSDCI calculations [21,26]. It should however be noted that the available EMS studies [24,25] are of poor energy resolution $(\sim 2.5 \mathrm{eV}$ fwhm). A high energy resolution EMS measurement of ethylene is currently in progress in our laboratory.

In summary, the issue of equivalence or non-equivalence of spectroscopic factors in EMS and PES is far from a closed issue. Interesting differences among theoretical studies, let alone between EMS and PES experiments, are still unresolved [4, 27-29]. Further experimental and theoretical studies are necessary and well-chosen benchmark systems such as ethylene will feature in the resolution of this important question. 


\subsection{XMPs and chemical reactivity}

A central problem in chemistry is the prediction of chemical reactions based on the knowledge of the electronic structure of the individual reactant molecules. Typical approaches to the investigation of chemical reactivity is the calculation of reaction indices such as the fukui index [30] or the decomposition of the intermolecular interaction energy into electrostatic, polarization and charge transfer contributions [31]. The goal of these theoretical approaches is to provide a rationalization for chemical empirical data on reaction rates such as acidity, basicity, proton affinity etc. If a "match" is achieved between the calculated fukui index, for example, and chemical empirical studies then a chemical rationalization is postulated. In most cases these studies have been most useful however they are prone to certain difficulties because typical "matches" are strongly dependent on the basis set employed in the calculation. For example in finding a chemical rationalization for the order of gas phase basicity in amines, Umeyama and Morokuma [31] used a 4-31G basis set and obtained the order of basicity: $\mathrm{NH}_{3}<R \mathrm{NH}_{2}<\mathrm{R}_{2} \mathrm{NH}<R_{3} \mathrm{~N}$ where $\mathrm{R}=\mathrm{CH}_{3}$. From an energy decomposition analysis they also found that the polarization energy contribution was the determining factor for the order of basicity. Using a STO-3G basis set the order of basicity was found to be the same as above $[32,33]$ but the energy decomposition analysis showed that the determining factor was the charge transfer contribution and not the polarization contribution. Therefore from the 4-31G studies one can rationalize the order of basicity as due to polarization whereas from the STO-3G studies one can rationalize the order of basicity as due to charge transfer.

Electron momentum spectroscopy can provide a resolution to this dilemma since the experimental momentum profiles (XMPs) obtained in EMS can be used to evaluate the quality of theoretical wavefunctions used in chemical rationalizations. In fact, this has been done for the order of basicity in the amines $[34,35]$. Thus a preliminary answer can be given to the question: Can XMPs be related to the reactivity of complex molecular systems? The answer is affirmative. Unfortunately the nature of the relation is not entirely clear since chemical reactivity is a broad subject and subtle factors such as solvent effects and steric effects are neglected. Furthermore, the accurate evaluation of theoretical wavefunctions using XMPs for complex molecular systems are dependent on several technical problems such as:

(i) the need for high energy resolution EMS;

(ii) the need for introducing complex molecular systems into the gas phase;

(iii) the need for high coincidence countrates;

(iv) the need for accurate understanding of the momentum resolution effects and the momentum scale.

Problem (iv) has been addressed in a series of simulation studies [36-38] wherein finite collision volume effects have been found to have a significant effect at low momentum. From the Fourier transform relation low momentum corresponds to the chemically sensitive region of the molecule and thus the region most useful in the evaluation of theoretical wavefunctions for chemical rationalizations. Figure 2 illustrates the dramatic effect of proper inclusion of momentum resolution effects in the comparison of the most accurate wavefunction for water and the measured XMP for the outermost orbital of water. Excellent agreement is obtained between experiment and theory. The remaining small discrepancy originally observed between experiment and theory by Bawagan et al. [39] has been eliminated. It should be noted that in this application (Fig. 2) the inclusion of momentum resolution effects, according to one view $[36,37]$, changes the momentum scale on which the XMP data points are placed. This new momentum scale is referred to as the average momentum scale and can be obtained by averaging a series of electron trajectories emanating from a finite collision volume (see upper panel of Fig. 2). An alternative approach is to place the XMP data points on the nominal momentum scale and incorporate the momentum resolution effects using appropriate instrumental resolution functions [38]. Although both approaches ultimately provide the same information, the 


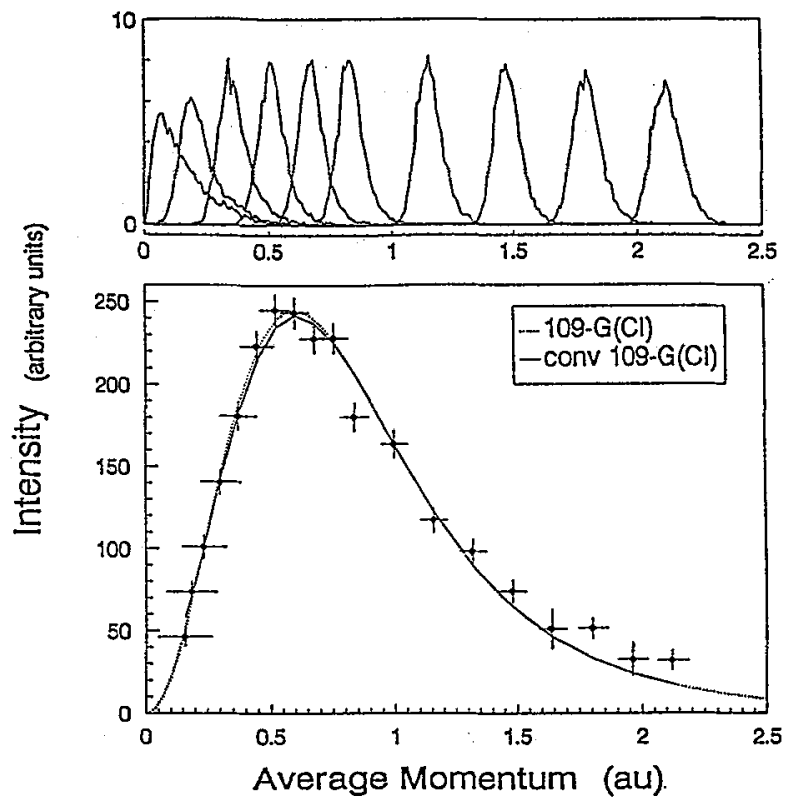

Figure 2: Lower panel: $\mathrm{H}_{2} \mathrm{O}_{1} \mathrm{~b}_{1} X M P$ (diamonds) and theoretical ion-neutral overlap distribution plotted on the average momentum scale [36,37]. The theoretical overlap distribution without resolution convolution (broken line) and with resolution convolution (solid line) are shown. Upper panel: Area normalized instrumental momentum resolution functions at different azimuthal angles [49]. The widths of the resolution functions are reflected on the horizontal error bars in the lower panel.

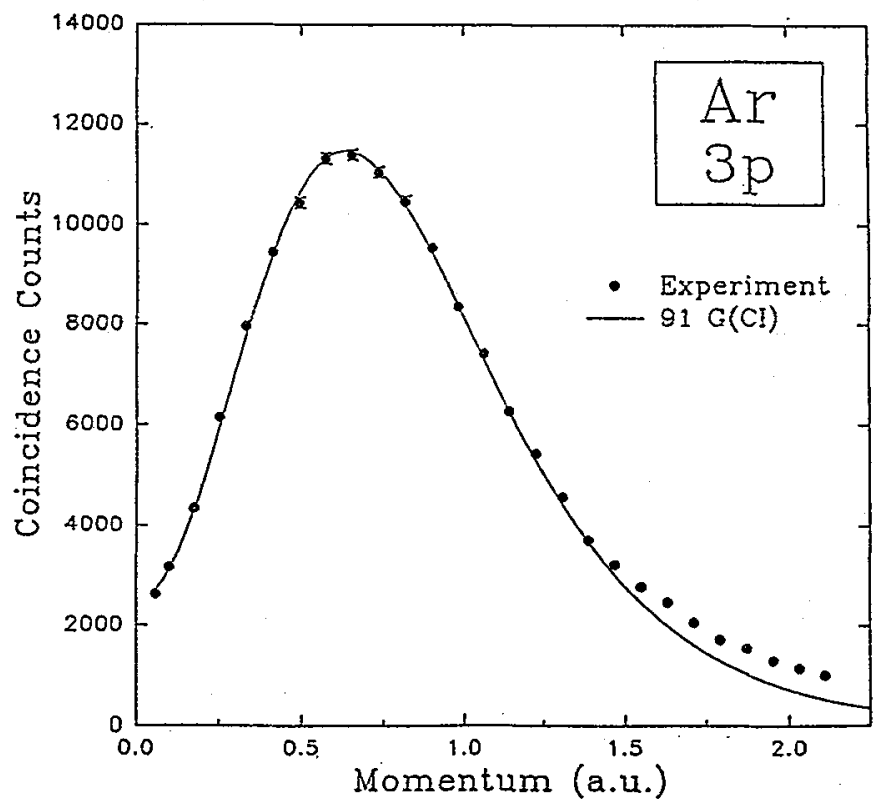

Figure 3: Argon 3p XMP data collected over 43 hours together with a resolution folded momentum profle using an MRSDCI wavefunction [43]. 
use of the average momentum scale $[36,37]$ is more appealing because of its self-consistency.

With an accurate understanding of momentum resolution effects, proper evaluation of theoretical wavefunctions can be made. This procedure has led to the successful design of so-called universal wavefunctions for $\mathrm{H}_{2} \mathrm{O}$ [39], $\mathrm{NH}_{3}$ [40], $\mathrm{H}_{2} \mathrm{~S}$ [41], $\mathrm{HF}$ and $\mathrm{HCl}$ [42]. These new wavefunctions are universal in the sense that most calculated molecular properties are within $1 \%$ of all experimental values. In other words, the universal wavefunctions are of "chemical quality".

Problem (iii) has recently been addressed successfully by Todd et al. [43]. A sample of the Ar 3p XMP obtained using their new multichannel momentum dispersive EMS spectrometer is shown in Figure 3 . The coincidence countrate reported with this new spectrometer is $\sim 600$ counts/minute which is an order of magnitude improvement compared to single channel EMS spectrometers. With significantly improved coincidence countrates, experimental momentum profiles of high accuracy can now be routinely obtained. The successful resolution of the above-mentioned technical problems are motivated by future practical chemical applications of EMS such as computer aided molecular design. The extension of the interactive experimental-theoretical evaluation of wavefunctions of small molecular systems to larger and more complex systems will surely be awaited.

\subsection{Complex molecular systems and $(e, 2 e)$ reaction theory}

Much of our understanding of the $(e, 2 \mathrm{e})$ reaction mechanism is based on two general experimental schemes namely,

(a) Under high momentum transfer conditions at high impact energies, the dependence of the XMPs on the impact energy indicate whether the plane wave impulse approximation (PWIA) is valid or not $[44,45]$;

(b) Under low momentum transfer conditions at intermediate and low impact energies, the angular distribution of the outgoing electrons indicate whether there is a need for including extra terms in the Born series for the scattering amplitude or not $[46,47]$.

A great majority of these (e,2e) reaction studies have concentrated on simple atomic systems and in a few cases on simple diatomic systems. In particular, at high impact energy and high momentum transfer the general validity of PWIA for molecular systems is generally assumed based on calibration studies with atomic systems. If we are to use EMS reliably in chemistry, further investigations of the impact energy dependence of molecular XMPs are necessary. The multicenter nature of the target presents a multiplicity of reaction channels such as dissociation, re-arrangements and autoionization which are not present or greatly reduced in atomic systems.

We have attempted to investigate the (e,2e) reaction mechanism in a slightly different way. Our EMS experiments under non-coplanar, symmetric scattering conditions at $E_{0}=1000 \mathrm{eV}$ provide enough information which we believe would allow us to accomplish this. We start with the following conjecture:

The correlation coefficient ( $\rho$ ) of the bivariate distribution of coincidences as a function of the binding energy and the time delay of the two outgoing electrons provides information on the (e,2e) reaction mechanism. If the (e,2e) reaction is dominated by impulsive, binary collisions then $\rho=0$ ie. the binding energy and time delay are statistically independent variables. If the (e,2e) reaction involves to a significant degree multiple collisions or reaction channels other than the impulsive, binary type then $\rho \neq 0$.

The distribution of coincidence counts is a function of two variables namely, the binding energy $\left(E_{b}\right)$ and the time delay $(\Delta t)$. The most common bivariate distribution [48] is the normal bivariate 


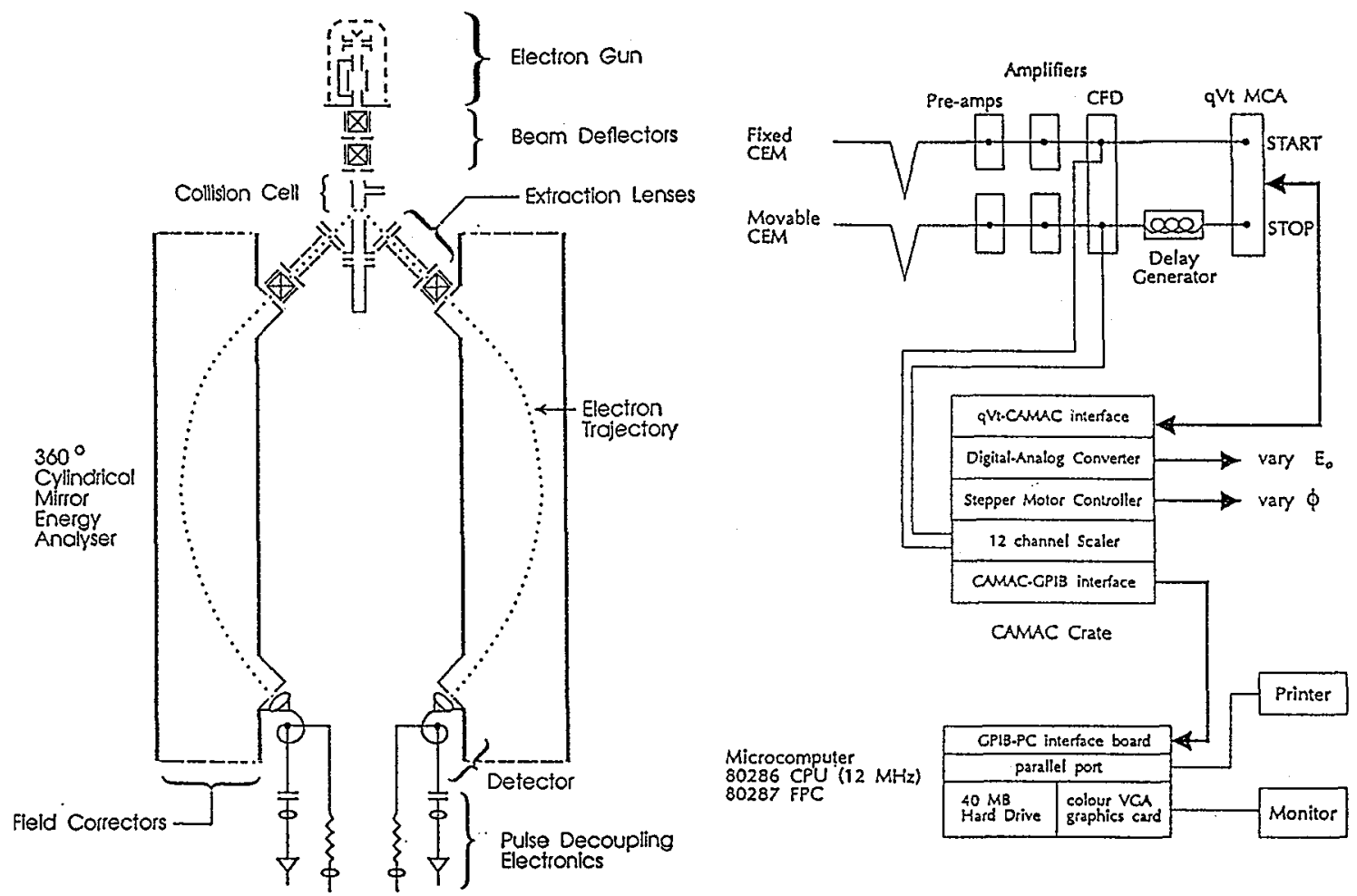

iigure 4: Coincidence electron momentum spectrometer utilizing a full $\left(360^{\circ}\right)$ cylindrical mirror inalyzer and schematic of coincidence electronics and computer controlled data acquisition system.

or normal two-dimensional distribution given by,

$$
\begin{aligned}
\mathrm{p}\left(\mathrm{E}_{\mathrm{b}}, \Delta \mathrm{t}\right)= & \frac{1}{2 \pi \sigma_{E b} \sigma_{\Delta t} \sqrt{1-\rho^{2}}} \exp \left\{-\frac{1}{2\left(1-\rho^{2}\right)}\right. \\
& {\left.\left[\frac{\left(E_{b}-m_{E b}\right)^{2}}{\sigma_{E b}^{2}}+\frac{\left(\Delta t-m_{\Delta t}\right)^{2}}{\sigma_{\Delta t}^{2}}-\frac{2 \rho\left(E_{b}-m_{E b}\right)\left(\Delta t-m_{\Delta t}\right)}{\sigma_{E b} \sigma_{\Delta t}}\right]\right\} }
\end{aligned}
$$

where $\mathrm{m}_{\mathrm{E}_{\mathrm{b}}}, \sigma_{\mathrm{E}_{\mathrm{b}}}, \mathrm{m}_{\Delta \mathrm{t}}$ and $\sigma_{\Delta \mathrm{t}}$ are the means and standard deviations of the distributions in $\mathrm{E}_{\mathrm{b}}$ and $\Delta t$, respectively. $\rho$ is called the "correlation coefficient" for fluctuations in $E_{\mathrm{b}}$ and $\Delta t$ and is given by,

$$
\rho=\frac{\left\langle\left(\mathrm{E}_{\mathrm{b}}-\mathrm{m}_{\mathrm{Eb}}\right)\left(\Delta \mathrm{t}-\mathrm{m}_{\Delta \mathrm{t}}\right)\right\rangle}{\sigma_{\mathrm{Eb}} \sigma_{\Delta \mathrm{t}}}
$$

$\rho$ measures the degree to which $\mathrm{E}_{\mathrm{b}}$ and $\Delta$ t fluctuate in a mutually dependent manner. If $\rho$ is zero then the normal bivariate distribution (Eqn. 2) can be written as a simple product of two one-dimensional normal distributions.

An EMS spectrometer and computer-controlled coincidence detection system [49] capable of measuring the correlation coefficient are shown in Figure 4. Figure 5 shows a contour diagram of the 

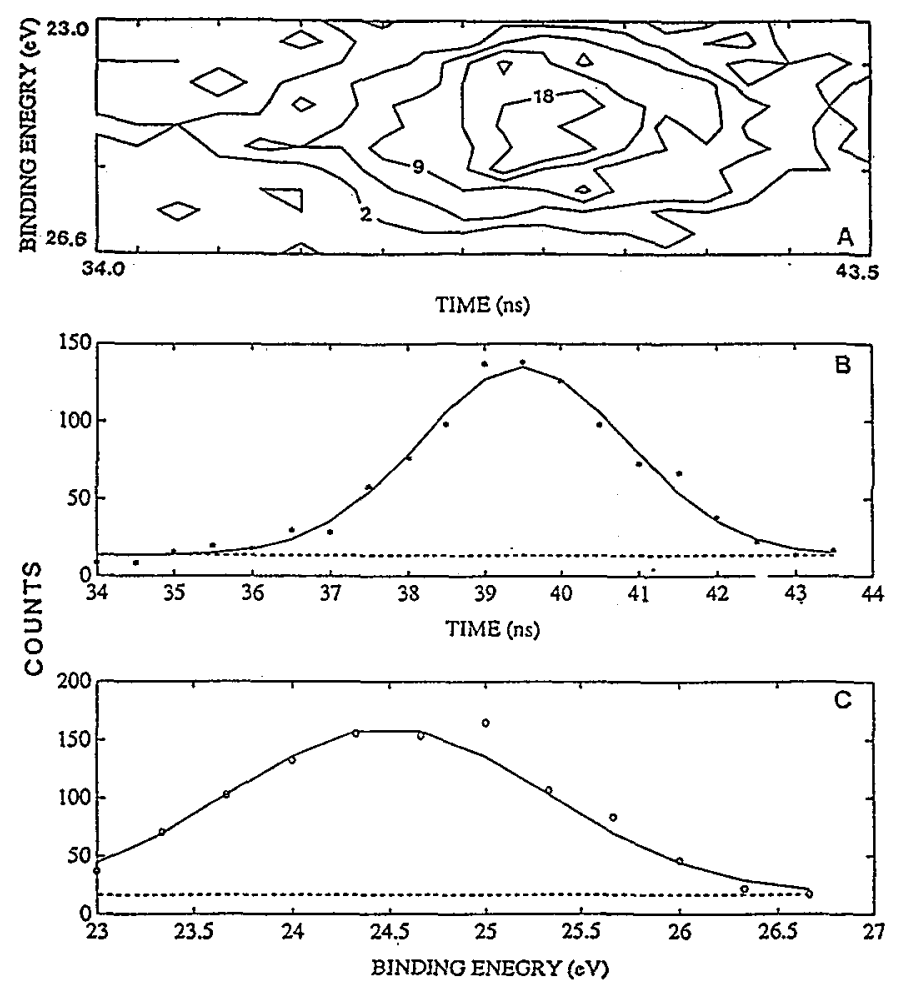

Figure 5: (a) Contour plot of coincidence peak in time delay spectrum of helium 1s orbital at $\mathrm{E}_{0}=$ $1000 \mathrm{eV}, \mathrm{E}_{\text {pass }}=100 \mathrm{eV}$ and $\phi \sim 0^{\circ}$. (b) Cumulative projection of the coincidence spectrum on the time delay axis. (c) Cumulative projection of the coincidence spectrum on the binding energy axis.

coincidence spectrum as a function of $\mathrm{E}_{\mathrm{b}}$ and $\Delta \mathrm{t}$ for the ionization of the helium 1s orbital at $1000 \mathrm{eV}$ impact energy and $100 \mathrm{eV}$ pass energy which are typical EMS operating conditions. The contour diagram (Fig. 5a) is clearly gaussian in shape as can be seen in the cumulative (summed) projections of the coincidence spectrum on both the time delay axis and the binding energy axis. Most of the previously reported EMS experiments do not save the total coincidence spectra thus an assessment of the correlation coefficient is not possible in these cases. It is generally assumed, however, that $\rho=$ 0 and the bivariate distribution is simply a product of two one-dimensional normal distributions in $\mathrm{E}_{\mathrm{b}}$ and $\Delta t$. The correlation coefficient for the coincidence spectrum shown in Fig. 5a, is calculated using Eqn. 3 and is found to be $\rho=0.05$ with an estimated uncertainty of \pm 0.10 [49].

A new procedure has been developed [50] to determine $\rho$ and its statistical uncertainty. The procedure involves the generation of a synthetic bivariate coincidence binding energy distribution using the known experimental energy and time resolutions. Several synthetic bivariate distributions with different assumed values of $\rho$ are generated and the Hessian summed difference [51],

$$
\sum_{i j} \frac{\left(M_{i j}^{e x p t}-M_{i j}^{t h e o}\right)^{2}}{\sigma_{i j}}
$$

is plotted as a function of $\rho$ using new experimental data [50] and shown in Figure 6. $M_{i j}^{\text {expt }}$ and $M_{i j}^{\text {theo }}$ are the $(i, j)$ element of the experimental coincidence binding energy distribution and the synthetic bivariate distribution, respectively. $\sigma_{i j}$ is the error involved in the $(\mathrm{i}, \mathrm{j})$ measurement. Note that the sum over $\mathrm{i}$ and $\mathrm{j}$ correspond to the binding energy and the time delay, respectively. 

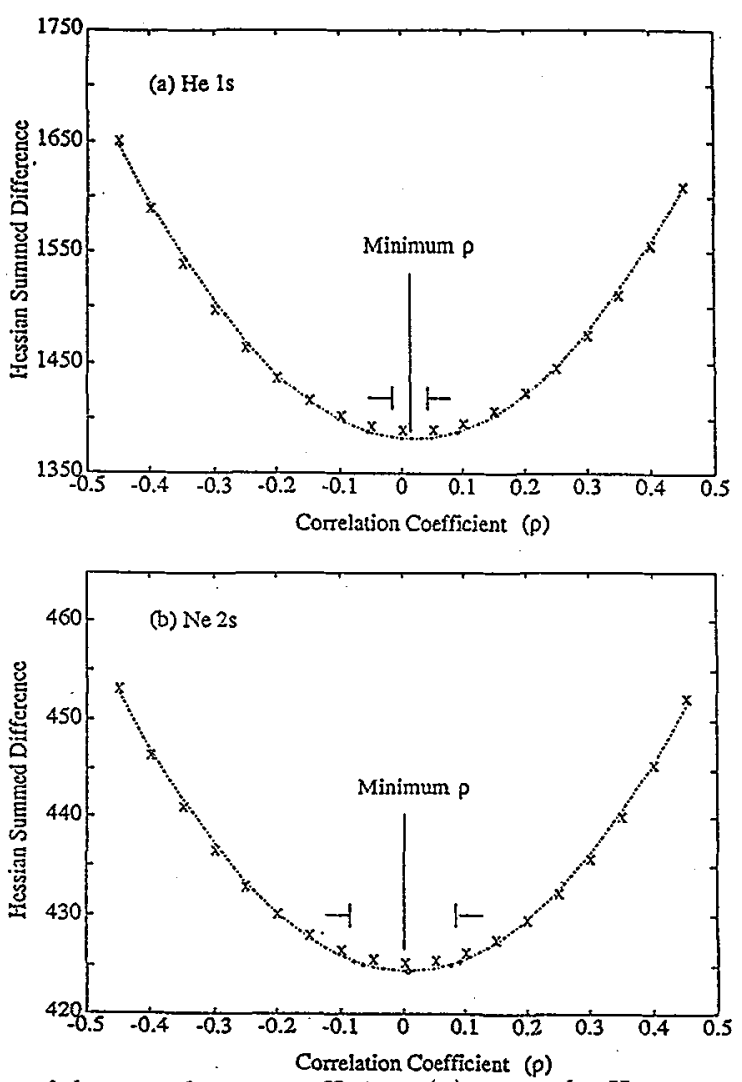

Figure 6: Determination of the correlation coefficient ( $\rho)$ using the Hessian summed difference method [50] for (a) $\mathrm{He} 1 \mathrm{~s}$ and (b) $\mathrm{Ne} 2 \mathrm{~s}$ coincidence binding energy spectra. The $\rho$ value is given by the minimum in the fitted poiynomial curve. The vertical bars indicate the uncertainty in $\rho$ obtained using the Hessian method.

The minimum in the curve (Fig. 6) corresponds to the $\rho$-value for the experimental coincidence distribution. The $\rho$-value obtained using this procedure is found to correspond exactly to that which can be calculated directly using Eqn. 3. An advantage of the present procedure is that it allows for the direct calculation of the uncertainty in $\rho$. The uncertainty in $\rho$ is illustrated by vertical bars in Fig. 6. The experimental correlation coefficient obtained for He 1s is $0.02 \pm 0.03$ which is a more accurate measurement than that reported recently [49]. A similar experiment has been made with the binding energy spectrum of $\mathrm{Ne} 2 \mathrm{~s}$ and is shown in Fig. 6b. The observed correlation coefficient for $\mathrm{Ne} 2 \mathrm{~s}$ is $0.02 \pm 0.09$. These results indicate that $\rho$ is effectively zero for both cases. Following the conjecture made earlier these results can be viewed as further experimental evidence that the PWIA is a valid framework for the interpretation of EMS experiments conducted at $E_{0}=1000 \mathrm{eV}$ under non-coplanar, symmetric geometry. The present results are not surprising since helium and neon have been thoroughly investigated using the impact energy dependence of the XMPs of the respective valence s-orbitals $[44,45]$. Furthermore, extensive studies at low and intermediate impact energies by Ehrhardt and co-workers [46] have indicated that first order theories are reliable for helium under high momentum transfer conditions.

Thus far, correlation coefficient studies have been limited to these simple systems but an important question to address is: If deviations from $\rho=0$ are detected, what do they precisely indicate? This is an important question and is the subject of current experimental and theoretical investigations. 
Our conjecture on $\rho$ and $(\mathrm{e}, 2 \mathrm{e})$ reaction mechanisms are motivated by practical and basic reasons. From a practical point of view, the correlation coefficient can be obtained (in principle) from every $(e, 2 e)$ experiment and thus available at no "extra cost". From a basic point of view, we believe the relation between $\rho$ and $(\mathrm{e}, 2 \mathrm{e})$ reaction mechanisms is plausible especially in the case of molecular $(e, 2 e)$ studies. In general, for molecular systems the binding energy peaks correspond to a manifold of unresolved vibrational states.

One can envision a correlation between binding energy and time delay in cases wherein two or more distinct scattering processes lead to final outgoing electron states similar to the final electron states in the $(e, 2 e)$ reaction. Since the final state of the ion is never observed in an $(e, 2 e)$ experiment, there are conceivably many other reaction channels other than that assumed in the plane wave impulse approximation (PWIA). For example, Avaldi et al. [52] have observed resonant channel effects in the asymmetric, coplanar $(e, 2 e)$ binding energy spectrum of acetylene. A statistically significant shift in the binding energy of the $1 \pi_{u}$ molecular orbital has been observed. It is likely that the correlation coefficient, if measured in this case, will also show deviations from $\rho=0$.

\section{Acknowledgements}

I would like to thank C.E. Brion for introducing me to the intricacies of EMS and for allowing me to reproduce Figure 3. I would also like to thank S. Owega, H. Luo and S. Desjardins for assistance in the preparation of this manuscript. This work was funded by the Natural Sciences and Engineering Research Council (NSERC) of Canada.

\section{References}

1. K.T. Leung and C.E. Brion, J. Elect. Spect. 35 (1985) 327.

2. K.T. Leung in Theoretical Models of Chemical Bonding, ed. Z.B. Maksic (Berlin, Springer, 1990).

3. I.E. McCarthy, J. Elect. Spect. 36 (1985) 37.

4. M. Ya. Amusia and A.S. Khiefets, Sov. Phys. JETP 59 (1984) 710.

5. R. Camilloni, A. Giardini-Guidoni, R. Tiribelli and G. Stephani, Phys. Rev. Lett. 29 (1972) 618.

6. E. Weigold, S.T. Hood and P.J.O. Teubner, Phys. Rev. Lett. 30 (1973) 475.

7. S.T. Hood, A. Hamnett and C.E. Brion, Chem. Phys. 39 (1976) 252.

8. C.E. Brion, A.O. Bawagan and K.H. Tan, Can. J. Chem. 66 (1988) 1877.

9. K.T. Leung and C.E. Brion, Chem. Phys. 82 (1983) 87.

10. I.E. McCarthy and E. Weigold, Phys. Rev. A31 (1985) 160.

11. H. Kossman, B. Krassig, V. Schmidt and J.E. Hansen, Phys. Rev. Lett. 58 (1987) 1620.

12. S. Svensson, K. Helenlund and U. Gelius, Phys. Rev. Lett. 58 (1987) 1624.

13. A.S. Khiefets, Sov. Phys. JETP 62 (1985) 260.

14. M.Ya. Amusia and A.S. Khiefets, J. Phys. B18 (1985) L679.

15. A.S. Khiefets and M. Ya. Amusia, Phys. Rev. A46. (192) 1261.

16. S. Braidwood, M. Brunger and E. Weigold, Phys. Rev. A47 (1993) 2927.

17. C.E. Brion, A.O. Bawagan and K.H. Tan, Chem. Phys. Lett. 134 (1987) 76.

18. I.E. McCarthy, R. Pascual, P. Storer and E. Weigold, Phys. Rev. A40 (1989) 3041.

19. A.D.O. Bawagan, B.J. Olsson, K.H. Tan, J.M. Chen and G.M. Bancroft, Chem. Phys. Lett. 179 (1991) 344.

20. A.D.O. Bawagan, B.J. Olsson, K.H. Tan, J.M. Chen and B.X. Yang, Chem. Phys. 164 (1992) 283. 
21. S.J. Desjardins, A.D.O. Bawagan and K.H. Tan, Chem. Phys. Lett. 196 (1992) 261.

22. S.J. Desjardins, Z.F. Liu, K.H. Tan, A.D.O. Bawagan, Y. Wang and E.R. Davidson (to be published).

23. B. Berndtsson, E. Basilier, U. Gelius, J. Hedman, M. Klassen, R. Nilsson, C. Nordling and S. Svensson, Physica Scipta 12 (1975) 235.

24. A.J. Dixon, S.T. Hood, E. Weigold and G.R.J. Williams, J. Electron Spectry. 14 (1978) 267.

25. M.A. Coplan, A.L. Migdall, J.H. Moore and J.A. Tossell, J. Am. Chem. Soc. 100 (1978) 5008.

26. C. Murray and E.R. Davidson, Chem. Phys. Lett. 190 (1992) 231.

27. A. Hibbert and J.E. Hansen, J. Phys. B20 (187) L245.

28. W. Wijesundera and H. Kelly, Phys. Rev. A36 (1987) 4539.

29. W. Wijesundera and H. Kelly, Phys. Rev. A39 (1989) 634.

30. K. Fukui, T. Yonezawa and C. Nagata, Bull. Chem. Soc. Japan 27 (1954) 423.

31. H. Umeyama and K. Morokuma, J. Am. Chem. Soc. 98 (1976) 4400.

32. W.J. Hehre and J.A. Pople, Tetrahedron Lett. 34 (1970) 2959.

33. A. Pullman and P. Brochen, Chem. Phys. Lett. 34 (1975) 7.

34. A.O. Bawagan, C.E. Brion, Chem. Phys. Lett. 137 (1987) 573.

35. A.O. Bawagan, C.E. Brion, Chem. Phys. 123 (1988) 51.

36. A.O. Bawagan and C.E. Brion, Chem. Phys. 144 (1990) 167.

37. L.A. Graham and A.D.O. Bawagan, Chem. Phys. Letters, 178 (1991) 441.

38. P. Duffy, C.E. Brion, M.E. Casida and D.P. Chong, Chem. Phys. 159 (1992) 347.

39. A.O. Bawagan, C.E. Brion, E.R. Davidson, and D. Feller, Chem. Phys. 113 (1987) 19.

40. A.O. Bawagan, R. Muller-Fiedler, C.E. Brion, E.R. Davidson and C. Boyle, Chem. Phys. 120 (1988) 335.

41. C.L. French, C.E. Brion and E.R. Davidson, Chem. Phys. 122 (1988) 247.

42. B.P. Hollebone, Y. Zheng, C.E. Brion, E.R. Davidson and D. Feller, Chem. Phys. 171 (1993) 303.

43. B.R. Todd, N. Lermer and C.E. Brion, Rev. Sci. Instrum. (subrnitted).

44. E. Weigold and I.E. McCarthy, Adv. At. and Mol. Phys. 14 (1978) 127.

45. I.E. McCarthy and E. Weigold, Rep. Prog. Phys. 54 (1991) 789.

46. H. Ehrhardt, K. Jung, G. Knoth and P. Schlemmer, Z. Phys. D1 (1986) 3.

47. A. Lahmam-Benani, H.F. Wellenstein, A. Duguet and A. Daoud, Phys. Rev. A30 (1984) 1511;

A. Lahmam-Benani, J. Phys. B24 (1991) 2401.

48. B.R. Frieden, Probability, Statistical Optics and Data Testing (Berlin, Springer-Verlag, 1990) p.53.

49. L.A. Graham, S.J. Desjardins and A.D.O. Bawagan, Can. J. Chem. 71 (1993) 216; L.A. Graham, MSc Thesis, Carleton University, 1991.

50. A.D.O. Bawagan, S. Owega and H. Luo, (to be published).

51. W.R. Leo, Techniques for Nuclear and Particle Physics Experiments (Springer-Verlag, Berlin, 1987) p. 104.

52. L. Avaldi, R. Camilloni, G.C. King and G. Stefani, Phys. Rev. A44 (1991) 4740. 